\title{
ESTUDO DA ADSORÇÃO/DESSORÇÃO DO ÁCIDO 2,4 DICLOROFENOXIACÉTICO (2,4D) EM SOLO NA AUSÊNCIA E PRESENÇA DE MATÉRIA ORGÂNICA
}

Eny Maria Vieira, Alexandre Gustavo Soares do Prado, Maria Diva Landgraf e Maria Olímpia de Oliveira Rezende Instituto de Química de São Carlos - Universidade de São Paulo - IQSC/USP - CP 780 - 13560-970 - São Carlos - SP

\begin{abstract}
STUDY OF ADSORPTION/DESORPTION OF HERBICIDE 2,4D IN SOIL. The herbicides are being used in huge quantities for various porpouses. Once the herbicide finds its way into the environment, a major part of it comes in contact with soil. Humic substances are major organic constituents of soil. These substances may interact with herbicides in different modes and adsorption is probably the most important one. Adsorption will control the quantity of herbicide in the soil solution, and determines its persistence, leaching, mobility and bioavailability. In this work we studied the interaction between the herbicide 2,4D and soil in the presence and absence of organic matter. The methodology utilized for the determination of 2,4D was gas chromatography with eletron capture detector. The behavior of $2,4 \mathrm{D}$ was evaluated through Freundlich isotherms. It was verified that the herbicide $2,4 \mathrm{D}$ has a large adsorption in the humic acid.
\end{abstract}

Keywords: humic substances; herbicide; 2,4D; adsorption; dessorption.

\section{INTRODUÇÃO}

Os objetivos primários de aumentar a colheita e melhorar a qualidade dos alimentos foram atingidos nos últimos anos, mas o uso indiscriminado sem os devidos critérios e sem conhecimento da ação e dos efeitos secundários por parte dos pesticidas pode acarretar danos ao meio ambiente e, conseqüentemente, a qualidade de vida do homem ficou comprometida.

Há vários fatores que contribuem direta e indiretamente para a redução da colheita agrícola. As ervas daninhas têm uma contribuição expressiva causando efeitos negativos na produção, uma vez que competem com a cultura por espaço, água, luz e principalmente nutrientes e muitas vezes são hospedeiras de pragas (insetos).

O solo é considerado como sendo um dos reservatórios finais dos herbicidas. Ao mesmo tempo ele é também fonte da qual os resíduos podem ser liberados para a atmosfera, lençol freático e organismos vivos.

O comportamento dos herbicidas no solo é bastante complexo e é resultante de vários fatores, sendo um deles a interação com os constituintes das diferentes frações contidas no solo (ácido húmico, ácido fúlvico, humina, argila, óxidos e etc.).

A adsorção é a interação do soluto (herbicida) da fase líquida com a superfície das partículas da fase sólida do solo. É um processo determinante para se entender o comportamento dos herbicidas, pois está relacionado diretamente aos processos de transporte, adsorção e bioatividade deste no solo, influenciando diretamente na disponibilidade dos produtos para as plantas e na ação seletiva dos herbicidas pela interferência no seu deslocamento.

\section{HERBICIDA ESTUDADO}

Neste estudo utilizou-se o herbicida ácido 2,4 diclorofenoxiacético $(2,4 \mathrm{D})$ grau técnico com $99 \%$ de pureza, procedente da Poly Science. O herbicida 2,4D é um ácido orgânico, com pKa 2,6, e possui uma solubilidade de $45 \mathrm{~g} / \mathrm{L}$ em água. Este herbicida é cancerígeno acarretando danos ao fígado e ao coração. Ataca o sistema nervoso central, provocando convulsões. Sua dose letal $\left(\mathrm{DL}_{50}\right)$ oral é de $370 \mathrm{mg} / \mathrm{kg}$ (em coelhos) e por via derme é de $1400 \mathrm{mg} / \mathrm{kg}$ (em camundongos).

Este herbicida tem ação de contato e é muito usado em plan- tações de trigo, arroz, milho, sorgo e principalmente cana de açúcar. Sua fórmula estrutural é apresentada na Figura $1^{1}$. O composto ácido 2,4 diclorofenoxiacético é comercializado com o nome de 2,4D. Seus sais e ésteres são os mais conhecidos agentes químicos utilizados como herbicida desta classe dos fenóxidos. Eles são usados pelos agricultores para controlar ervas daninhas de folhas largas. É usado também para controlar o mesmo tipo de ervas em acostamentos de estradas e sob linhas de transmissão elétrica ${ }^{2-4}$

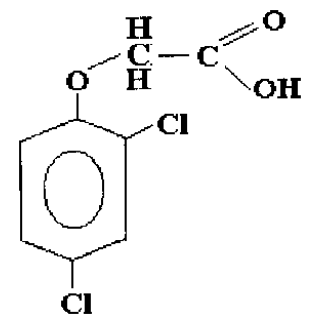

Figura 1. Fórmula Estrutural do Herbicida 2,4 D (Ácido 2,4 Dicloro fenoxiacético).

\section{PROCESSOS FÍSICO-QUíMICOS QUE AFETAM O PESTICIDA NO SOLO}

O comportamento dos pesticidas no solo pode ser influenciado por alguns fatores tais como: adsorção, movimento e decomposição. A adsorção influencia diretamente a magnitude do efeito de outros fatores como biodegradabilidade, bioacumulação e outros.

A adsorção é considerada como sendo um dos principais processos que afetam a interação que ocorre entre o pesticida e a fase sólida do solo. Os principais constituintes que representam a fase sólida no solo são: argila, minerais, matéria orgânica, óxidos e hidróxidos de alumínio e de ferro e sílica.

O movimento dos pesticidas no solo pode ocorrer por lixiviação, escoamento superficial e volatilização. As informações sobre o movimento de pesticidas são úteis para se fazer uma previsão da sua eficácia química. Os processos de decomposição operam um importante desempenho na dissipação de muitos pesticidas no solo. O desaparecimento de um pesticida do solo pode também se dar por vários processos químicos, inclusive fotodecomposição e reações químicas ${ }^{5,6}$. 
Os pesticidas estão sujeitos a interações que interferem na sua bioatividade, biodegradação e mobilidade no ambiente. A natureza das interações depende da composição do solo ${ }^{7}$.

\section{ISOTERMA DE ADSORÇÃO}

Vários modelos matemáticos de isoterma que descrevem a relação de adsorção para um pesticida no solo e seus constituintes são discutidos na literatura ${ }^{5}$.

O modelo de Freundlich tem sido largamente utilizado porque garante boa linearidade para solos ${ }^{5}$.

A forma da equação de Freundlich é apresentada na equação 1

$\mathrm{X} / \mathrm{M}=\mathrm{Kf} \cdot \mathrm{Ce} \mathrm{e}^{1 / \mathrm{n}}$

na qual $\mathrm{K}_{\mathrm{f}}$ é a constante de Freundlich e 1/n uma constante (índice da intensidade da adsorção) que depende da substância adsorvida e do meio adsorvente. Os valores de $K_{f}$ e $1 / n$ são determinados usando regressão linear na forma logarítmica da equação de Freundlich:

$\log \mathrm{X} / \mathrm{M}=\log \mathrm{K}_{\mathrm{f}}+(1 / \mathrm{n}) \log \mathrm{Ce}$

Tanto $\mathrm{K}_{\mathrm{f}}$ como $1 / \mathrm{n}$ são parâmetros de regressão característicos para cada sistema solo-herbicida. $\mathrm{O}$ parâmetro $\mathrm{X}$ representa o volume da solução de herbicida adicionado $(\mathrm{mL})$ e $\mathrm{M}$ é a massa do solo ( $\mathrm{g})$, Ce é a concentração do herbicida no equilíbrio com o solo $(\mathrm{mg} / \mathrm{mL})$.

Para as condições experimentais estudadas, $K_{f}$ e $1 / n$ são conhecidos como fator de capacidade e intensidade de adsorção respectivamente. $\mathrm{O} \mathrm{K}_{\mathrm{f}}$ é a quantidade de pesticida adsorvido quando a concentração no equilíbrio é igual a unidade. Assim, a medida da capacidade de adsorção $K_{f}$ e $1 / n$ indicam que a razão de adsorção aumenta com a concentração do soluto, refletindo na não linearidade da adsorção.

A isoterma de dessorção representa a relação entre a quantidade de um herbicida ainda remanescente no solo e em outros substratos, após o processo de dessorção, e a quantidade liberada para a solução aquosa, originalmente sem o composto após o equilíbrio a uma dada temperatura.

Muitos pesquisadores obtiveram alta correlação entre o teor de matéria orgânica e o coeficiente $\mathrm{Kd}^{8}$, sendo portanto um índice de grande utilidade na prática agrícola. O coeficiente de adsorção $(\mathrm{Kd})$ é calculado pela relação entre a quantidade de herbicida adsorvido no solo e a quantidade de herbicida na água em equilíbrio. Considerando que a fração da matéria orgânica dos solos é a principal responsável pela adsorção, podese obter um índice (Kom), dividindo-se (Kd) pelo teor de matéria orgânica (\% de matéria orgânica - OM)

$\mathrm{K}_{\mathrm{om}}=\frac{\mathrm{Kd}}{\% \mathrm{OM}} 100$

em que $\mathrm{K}_{\mathrm{om}}(\mathrm{L} / \mathrm{kg})$ é o coeficiente de distribuição de uma dada substância por unidade de matéria orgânica.

A matéria orgânica do solo estudado ${ }^{9}$ é composta de cerca de $58 \%$ de carbono orgânico (OC) em média (a quantidade de carbono orgânico nos solos varia de 52 a $65 \%$, ou seja $\mathrm{OC}=\mathrm{OM} \times 1,724)$. A partir desses valores pode-se calcular a constante Koc como descrito na equação $4^{9}$.

A adsorção de um herbicida pode ser normalmente obtida através das características do solo e é calculada pela constante de adsorção (Koc), baseada na quantidade de carbono orgânico contidas no referido solo ${ }^{10}$.
$\mathrm{K}_{\mathrm{oc}}=\left(\mathrm{K}_{\mathrm{d}} 100\right) /(\%$ de carbono orgânico $)$

$\mathrm{K}_{\mathrm{oc}}$ é também conhecido como coeficiente de distribuição de uma dada substância por unidade de carbono orgânico usado para comparar adsorções relativas de pesticidas em solo ${ }^{10}$.

\section{MATERIAIS E MÉTODOS}

O herbicida 2,4D foi preparado em solução estoque de 100 $\mathrm{mg} / \mathrm{L}$ em metanol. Os padrões nas concentrações utilizadas foram obtidos por diluição a partir da solução estoque.

As análises quantitativas foram feitas em um cromatógrafo a gás HP 5890 com detector de captura de elétrons, coluna capilar de sílica fundida (SPB-20) (20\% difenil, $80 \%$ dimetil polissiloxano entrecruzada) de $30 \mathrm{~m}$ de comprimento, $0,25 \mathrm{~mm}$ de d.i. e $0,25 \mu \mathrm{m}$ de espessura de filme. Temperatura da coluna, detector e injetor $220^{\circ} \mathrm{C}$ e $280^{\circ} \mathrm{C}$ respectivamente. Rampa de aquecimento de $5^{\circ} \mathrm{C}$, split de $1: 25$ volume de injeção $1 \mu \mathrm{L}$, fluxo do gás (nitrogênio) e velocidade linear $1 \mathrm{~mL} / \mathrm{min}$.

\section{Adsorção do herbicida 2,4D no solo na ausência e na presença de matéria orgânica}

Foram colocadas 27 soluções padrões do herbicida nas concentrações de 0,2 a $60 \mathrm{mg} / \mathrm{L}$ em erlenmeyers de $125 \mathrm{~mL}$ e deixou-se evaporar até à secura, após adicionou-se $0,01 \mathrm{~g}$ de cloreto de cálcio, $1,0 \mathrm{~g}$ de solo com matéria orgânica e $10 \mathrm{~mL}$ de água e deixou-se sob agitação por 30 minutos à temperatura ambiente, após acidificou a pH 2,0, centrifugou-se por $20 \mathrm{~min}$. a $3.000 \mathrm{rpm}$, fez-se a extração do sobrenadante com $3 \times 10 \mathrm{~mL}$ de éter etílico, deixou-se evaporar até a secura, ressuspendeuse com 2,0 mL de metanol metilou-se com diazometano e determinou-se por GC/ECD. Com a fração sólida foi feita a dessorção com a adição de $10 \mathrm{~mL}$ de água e procedendo da mesma forma da adsorção. O mesmo procedimento foi adotado para o solo livre de matéria orgânica.

\section{RESULTADOS E DISCUSSÃO}

A Tabela 1 apresenta as características do solo da Fazenda Experimental do Instituto Agronômico de Campinas em Pindorama $\mathrm{SP}$, determinadas por métodos físicos e físico-químicos.

Com o objetivo de se conhecer o tempo em que o sistema atinge o equilíbrio, determinou-se o tempo mínimo de adsorção para ambos os sistemas de solo com e livre de matéria orgânica.

A Figura 2 representa a concentração de 2,4D adsorvida no solo com matéria orgânica em função do tempo.

Ao observar o comportamento do sistema herbicida-solo com matéria orgânica, quanto ao tempo de adsorção. Verificase que o equilíbrio foi atingido por volta de 25 minutos então optou-se por fazer a adsorção em 30 minutos.

Analisando o sistema herbicida-solo livre de matéria orgânica, verifica-se que o tempo de adsorção atingiu o equilíbrio a cerca de 25 minutos, neste caso também optou-se por fazer a adsorção em 30 minutos.

A partir dos valores obtidos da adsorção do herbicida 2,4D no solo, na presença e na ausência de matéria orgânica, por grama do solo, e a concentração deste herbicida presente na solução após 30 minutos (Ce), obtém-se os gráficos que são apresentados nas figuras 4 e 5 .

Tabela 1. Características do Solo de Pindorama (9).

\begin{tabular}{ccccccc}
\hline Solo & MO $(\%)$ & Areia $(\%)$ & Silte $(\%)$ & Argila(\%) & Tipo Argila & pH \\
\hline Pindorama & 0,7 & 69 & 12 & 19 & C,G,M,V & 6,9 \\
\hline
\end{tabular}

(MO = matéria orgânica, $\mathrm{C}=$ Caulinita, $\mathrm{G}=$ Gipsita, $\mathrm{M}=$ Mica, $\mathrm{V}=$ Vermiculita $)$. 


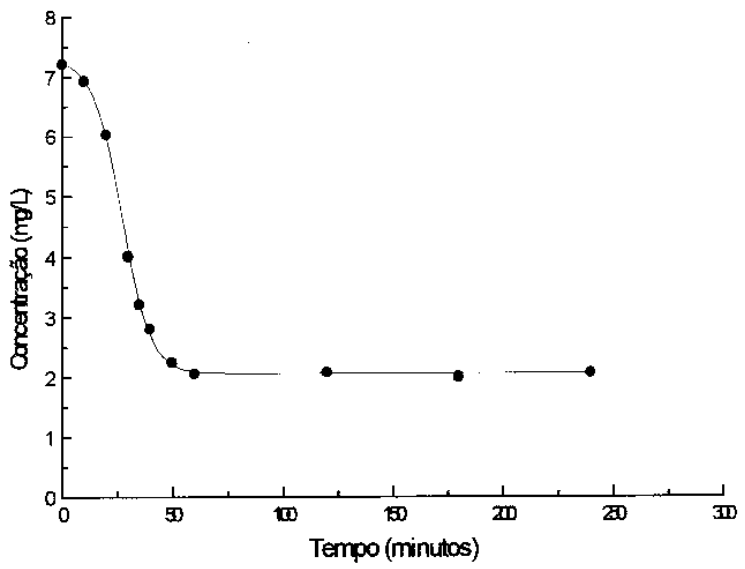

Figura 2. Tempo de Adsorção do Herbicida 2,4D no Solo com Matéria Orgânica.

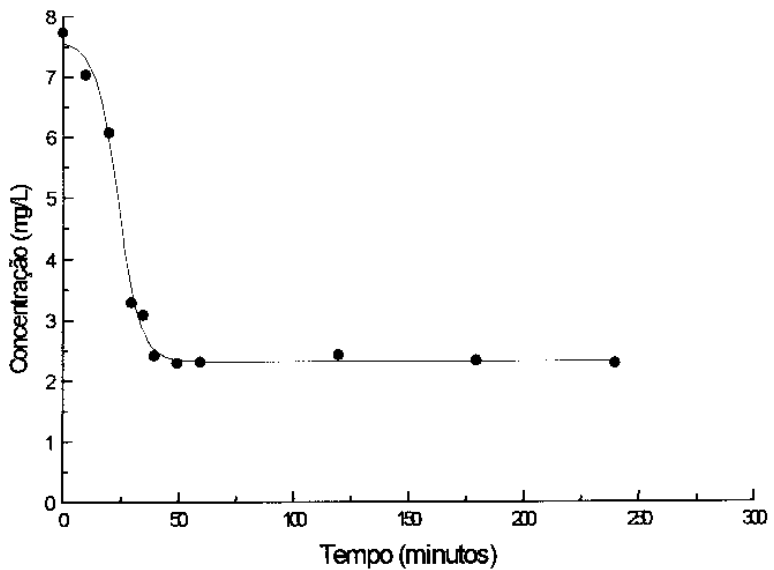

Figura 3. Tempo de Adsorção do Herbicida 2,4D no Solo Livre de Matéria Orgânica (Calcinado).

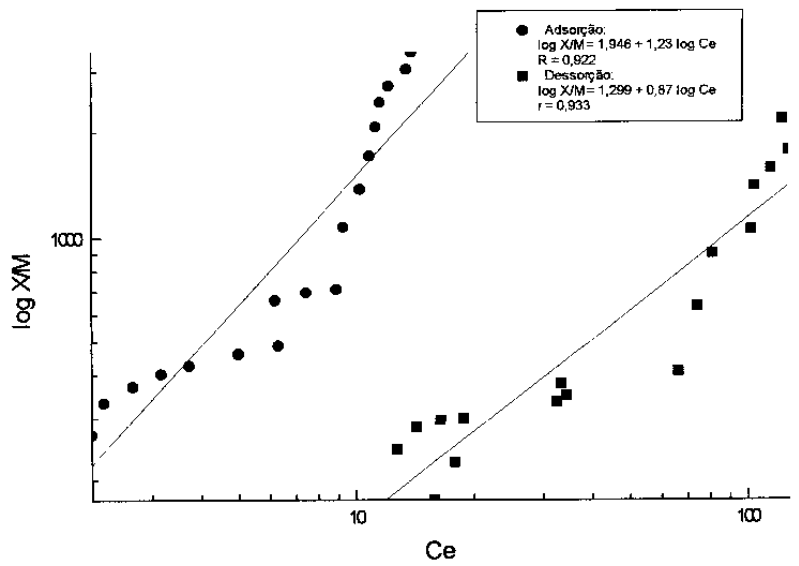

Figura 4. Isoterma de Freundlich de Adsorção e Dessorção do 2,4D no Solo sem Calcinar (com Matéria Orgânica).

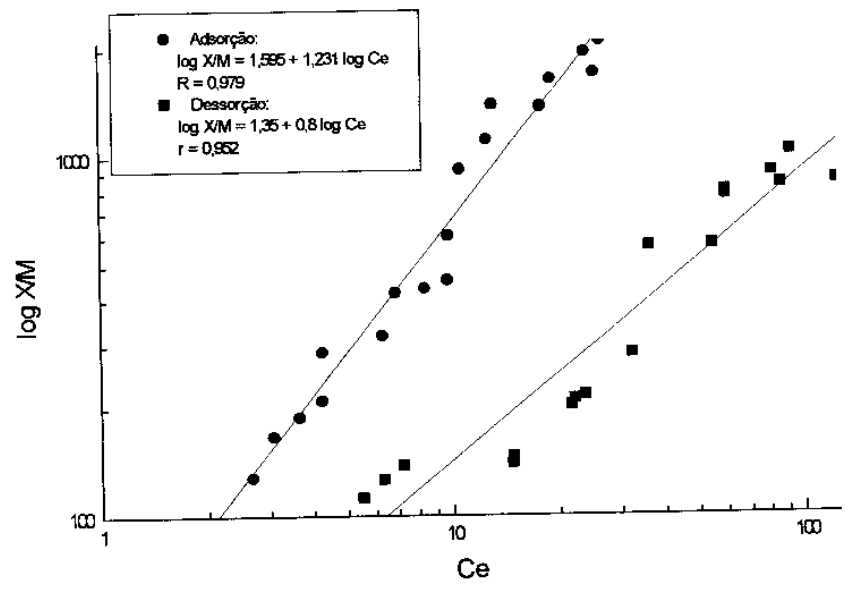

Figura 5. Isoterma de Freundlich de Adsorção e Dessorção do 2,4D no Solo Calcinado (Livre de Matéria Orgânica).

Tabela 2. Constantes de Freundlich calculadas a partir dos gráficos.

\begin{tabular}{lcccc}
\hline & \multicolumn{2}{c}{ ADSORÇÃO } & Kf & DESSORÇÃO \\
\hline & Kf & $1 / \mathrm{n}$ & $22,16 \pm 0,23$ & $0,80 \pm 0,05$ \\
\hline Solo calcinado & $39,28 \pm 0,30$ & $1,23 \pm 0,09$ & $20,12 \pm 0,03$ & $0,87 \pm 0,05$ \\
Solo com MO & $88,48 \pm 0,08$ & $1,23 \pm 0,07$ & &
\end{tabular}

MO = matéria orgânica

A Tabela 2 apresenta os valores de $K_{f}$ de adsorção/ dessorção para o 2,4D no solo na presença e na ausência de matéria orgânica.

As diferentes estruturas de biopolímeros contidos nas plantas que dão origem à matéria orgânica, podem estar presentes em algumas superfícies ativas contribuindo para o processo de adsorção de herbicidas no solo.

Efeitos típicos do processo de humificação da matéria orgânica são: aumento de suas propriedades coloidais e a incorporação de uma variedade de grupos funcionais de diferentes reatividades.

$\mathrm{Na}$ presença da matéria orgânica, compostos orgânicos simples (como exemplo o herbicida 2,4D), podem promover uma variedade de interações sortivas através de equilíbrio reversível. Esses processos também podem afetar a intensidade de bioatividade e lixiviação por parte destes compostos no ambiente.

A adsorção nos colóides do solo (tanto orgânico como inorgânico) especificamente na fração mineral, para a maioria dos pesticidas envolve diferentes mecanismos, tais como: fixação física, força de van der Waals, ligação de hidrogênio, ligação iônica, complexação através de íons metálicos, ligação hidrofóbica, complexo de transferência de carga, interações eletrostáticas e ligações covalentes também são possíveis.

Como se pode observar pelos valores de $\mathrm{K}_{\mathrm{f}}$ na Tabela 2, a adsorção do 2,4D no solo livre de matéria orgânica foi relativamente baixa quando comparada com a do solo contendo matéria orgânica, o que havia sido constatado em pesquisas anteriormente realizadas ${ }^{11}$.

Analisando os valores de $\mathrm{K}_{\mathrm{f}}$ de dessorção, tanto no solo calcinado como no solo com matéria orgânica, verifica-se que no caso do solo calcinado o herbicida dessorve muito, tendo uma pequena quantidade remanescente na matriz do solo.

Quanto ao modelo de isoterma sugerido podemos observar que quando na presença de matéria orgânica a interação entre o herbicida 2,4D e o solo é bastante forte até aproximadamente $10 \mathrm{mg} / \mathrm{mL}$ de 2,4D. Após essa concentração a interação é mais fraca, denotando a presença de outro sítio de interação. 
Tabela 3. Valores de Kd, Kom e Koc.

\begin{tabular}{lccc}
\hline Amostra & Kd & Kom & Koc \\
\hline Solo calcinado & $304,12 \pm 1,02$ & - & - \\
Solo com MO & $361,16 \pm 1,07$ & $51.594,00 \pm 6,82$ & $88.948,05 \pm 9,04$ \\
\hline
\end{tabular}

MO = matéria orgânica

É possível que uma pequena parte do 2,4D tenha adsorvido irreversivelmente na matriz do solo, tanto no solo com matéria orgânica como livre da mesma.

A baixa adsorção do 2,4D no solo pode ser atribuída ao fato de que as cargas negativas dos colóides do solo repelem o herbicida conforme citado na literatura ${ }^{12}$. Isto pode ocorrer porque o 2,4D quando está em solução aquosa encontra-se na forma aniônica, portanto carregado negativamente, nestas condições o herbicida não tem afinidade por superfícies negativas, como por exemplo argilas .

São claras as evidências de que o herbicida 2,4D não adsorve muito nas argilas, este fato pode ser observado pelos resultados da adsorção no solo calcinado (Tabela 2).

De acordo com dados obtidos, verifica-se que o teor de matéria orgânica no solo é um fator muito importante, quando se trata de contaminantes ambientais tais como herbicidas e metais pesados isto pode ser observado segundo os valores de $\mathrm{K}_{\mathrm{f}}$ para o solo contendo matéria orgânica.

Thompson et al ${ }^{13}$ estudaram a persistência do herbicida $2,4 \mathrm{D}$ em solos e verificaram que a lixiviação deste herbicida foi menor em solos com alto teor de matéria orgânica e baixo valor de $\mathrm{pH}$, características estas que têm grande influência na adsorção de herbicidas ácidos, então há maior persistência do 2,4D no solo. Isto se deve ao fato de que o herbicida 2,4D tem grande adsorção na matéria orgânica e ele perde sua ação, mas pode ficar adsorvido nas substâncias orgânicas sem se degradar.

A Tabela 3 apresenta os valores do coeficiente de adsorção $\mathrm{Kd}$, coeficiente de distribuição $\left(\mathrm{K}_{\mathrm{om}}\right)$ do $2,4 \mathrm{D}$ por unidade de matéria orgânica e coeficiente de distribuição (Koc) do 2,4D por unidade de carbono orgânico.

\section{CONCLUSÕES}

Analisando os valores de Kf (coeficiente de adsorção) encontrados do 2,4D no solo com e livre da metéria orgânica, pode-se dizer que este herbicida adsorve preferencialmente na matéria orgânica.
Os $\mathrm{K}_{\mathrm{f}}$ de dessorção tanto para o solo com matéria Orgânica como livre desta observa-se que são bem próximos, isso nos leva a crer que nos dois sistemas dificilmente o $2,4 \mathrm{D}$ será lixiviado para outras camadas do solo e mesmo para o lençol freático.

\section{REFERÊNCIAS}

1. The Agrochemical Handbook Notthingham. The Royal Societ of Chemistry 1987. p. A 111 - 2.

2. Ottaway, J. H.; Bioquímica da Poluição. Edusp, São Paulo 1982 ; p 3.

3. Kearney, P. C.; Kaufman, D. D.; Herbicides chemistry degradation and mode of action. Marcel Dekker, New York 1976; p 1.

4. Hatzios, K. K.; Hoagland, R. E. Crop safners for herbicides. Academic Press, New York 1988; p 10.

5. Tavares, M. C. H.; Dissertação de Mestrado, Instituto de Química de São Carlos; Universidade de São Paulo, São Carlos. 1995.

6. Bailley, G. W.; White, J.; J. Agr. Food Chem. 1964, 12, 324.

7. Weber, J. B.; Miller, C. T. In Reaction and movement of organic chemical in soil; Sawaney, B. L.; Brown, K., Ed., SSSA, Madison 1991; p 63.

8. Francioso, O.; Bak, E.; Rossin, N.; Saqui, P.; The Sci. of the total Environ. 1992, 123/124, 503.

9. Vaz, C. M. P.; Tese de Doutorado - Centro de Energia Nuclear na Agricultura: Universidade de São Paulo. Piracicaba. 1994

10. Mccall, P. J.; Swann, R. L.; Laskowski, D. A.; Unger, S. M.; Vrona, S. A.; Dishburger, H. J.; Bull. Environ Contam. Toxical. 1980, 24, 190.

11. Schnitzer, M. M.; Khan, S. U.; Soil Organic Matter; Elsevier, New York 1978; 136.

12. Groves, R.; Smith, A. E.; Can. J. of Soil Sci. 1974, 54, 179.

13. Thompson, D. G.; Stephenson, G. R.; Solomon, K. R.; Skepasts, A V.; J. of Agric and Food Chem. 1984, 32, 578. 